\title{
LA VIOLENCIA INVISIBLE DE LO COTIDIANO Y RESPONSABILIDAD DEL PROFESIONAL EN CIENCIAS SOCIALES
}

\author{
América Celeste Guevara Parra
}

\author{
Universidad Autónoma de Bucaramanga, Colombia
}

\begin{abstract}
Resumen: El objetivo de este escrito es reflexionar en torno a la indiferencia propia de un mundo regido por el interés personal donde todo ha sido permeado por la lógica productiva, incluso las relaciones entre los seres humanos. La organización del ensayo se estructura en tres apartados correspondientes a los subtemas principales: la violencia cotidiana hacia grupos socialmente desfavorecidos; el discurso de felicidad ligado a la lógica de la productividad como imperativo de la época; y una pregunta por la posición desde la que intervienen los profesionales en ciencias sociales. Se acude al "Método analítico" como herramienta metodológica pues invita a introducir y mantener el cuestionamiento respecto al tema de interés a partir de un saber siempre por constituirse, más que a refugiarse en la comodidad de caminos metodológicos prestablecidos que no dan lugar ni a la sorpresa ni al deseo del investigador. A modo de conclusión, se menciona la incidencia de nuevas formas de agresión que proliferan de manera global, silenciosa y constante en forma de xenofobia, racismo y clasismo hacia sectores a los que les es difícil adaptarse al sistema productivo y se destaca la función de las ciencias sociales como facilitadoras de recursos simbólicos necesarios para tramitar el malestar contemporáneo en sujetos y comunidades que han sido desechados por el sistema.
\end{abstract}

Palabras Clave: violencia transpolítica, posmodernidad, pensamiento positivo, intervención social, recursos simbólicos.

\begin{abstract}
The objective of this paper is to reflect on the indifference of a world governed by personal interest where everything has been permeated by the logic of production, including the relationships between human beings. The organization of the essay is structured in three sections corresponding to the main sub-themes: daily violence towards socially disadvantaged groups; the discourse of happiness linked to the logic of productivity as an imperative of the times; and a question about the position from which social science professionals intervene. The "Analytical method" is used as a methodological tool because it invites to introduce and maintain the questioning of the subject of interest from a knowledge always to be constituted, rather than taking refuge in the comfort of preset methodological paths that give no room for surprise or for the researcher's desire. In conclusion, mention is made of the incidence of new forms of aggression that proliferate globally, silently and constantly in the form of xenophobia, racism and classism towards sectors that find it difficult to adapt to the productive system, and it is emphasized in the role of the social sciences as facilitators of the symbolic resources needed to deal with contemporary discomfort in subjects and communities that have been discarded by the system.
\end{abstract}

Keywords: Transpolitical Violence, Postmodernity, Positive Thinking, Social Intervention, Symbolic Resources. 
La violencia invisible de lo cotidiano y responsabilidad del profesional en ciencias sociales

\section{Introducción}

El presente texto reúne reflexiones que han sido articuladas a partir del trabajo y discusiones de varios años al interior del grupo de investigación "Violencia, Lenguaje y Estudios Culturales" de la Universidad Autónoma de Bucaramanga (Colombia) en torno a problemáticas relacionadas especialmente con la violencia, el duelo y la memoria histórica. El título da cuenta de una noción, la Violencia Transpolítica, que ha sido objeto de análisis constante en el grupo mencionado y es tomada de la obra del filósofo francés Jean Baudrillard quien la conceptualiza como una forma de violencia propia de esta época caracterizada tanto por el exceso en el consumo, como por la proliferación de las comunicaciones donde todo circula como un producto. Es decir, ya no se trata solo de la violencia externa típica de las guerras, sino de aquella caracterizada por el anonimato y el silencio de agresiones socialmente veladas (y toleradas) hacia quienes no se ajustan al imperativo de productividad. Este concepto de Violencia cotidiana o transpolítica se desarrolla en el primer apartado y es puesto en relación con el escenario de la posmodernidad en el que acontece. En un segundo apartado se presenta, a partir de la obra de Ehrenreich sobre el modelo de Pensamiento Positivo, los efectos de la incorporación de cierta idea de felicidad ligada a ser empresario de sí mismo, y se hace mención a las consecuencias que esto implica para sujetos que son producidos como desechos. En el tercer apartado, a través de posturas como las de Lyotard, Baudrillard, Butler y desde el psicoanálisis Lacan y Recalcati, se cuestionan modos de proceder en las disciplinas sociales que, por medio de esquemas metodológicos preestablecidos orientados a la estandarización del saber, privilegian el mantenimiento del poder en manos de los autorizados, "los expertos" y, en esta vía, no diferencian entre la producción de sentido y la imposición de un sentido en los sujetos y comunidades con las que trabajan. Finalmente, cabe anotar que el proceder metodológico aquí privilegiado es afín al "Método analítico", concebido por el psicoanálisis que entiende el método como una actitud desprejuiciada, crítica y pluralista y no como un algoritmo cuya puesta en práctica asegura la solución de un problema determinado. Una vez descubierto un algoritmo otros pueden aplicarlo sin necesidad de conocer los fundamentos del mismo (Lopera, 2010), el método analítico, por su parte, se trata no de un conjunto articulado de conocimientos, sino de recorrer un camino a partir de un saber por constituirse y del gusto por trabajar para producir ese saber, lo que supone no tener todo resuelto, así como ver más preguntas que respuestas. Por eso, más que lineamientos específicos a los profesionales en ciencias sociales, se plantean interrogantes sobre diferentes formas de proceder que, en su afán de considerarse universalmente validas, no cuentan con el otro, el sujeto con el que trabajan. 


\section{Posmodernidad y Violencia Transpolítica}

A Colombia "se le juzga el país sin estado de guerra abierta más violento del mundo" (Daza - Zuela, 2017: 105). No obstante, los ciudadanos de esta nación no sólo ignoramos o somos indiferentes respecto al pasado político violento del país, sino que, al no empuñar un fusil, somos incapaces de pensarnos como copartícipes de nuestras violencias. Predomina la idea "aquí no se puede hacer nada" (Mojica, 2015: 150) pues se soslaya que en la cotidianidad accionamos armas crueles expresadas en el maltrato al más próximo, en la segregación social, el clasismo, el racismo y la discriminación. Dicha violencia constante y de baja intensidad que surge desde lo cotidiano en la relación con los otros (también conocida como violencia transpolítica) no se desliga de las lógicas que ayudan a exacerbar y mantener el conflicto armado interno más largo del mundo. En el presente texto, lo anterior será analizado a la luz de un conjunto de factores que golpean a las subjetividades de nuestra época, conceptualizada por teóricos como Bauman, Lyotard, Baudrillard o Lipovestky como hipermodernidad (Montesinos, 2002). Es decir, la modernidad llevada a su punto de máxima realización resultado de la liberación en todos los campos, en lo político, social, sexual, artístico. Pero todo lo que ha sido liberado lo ha sido para ser puesto en circulación como mercancía (Baudrillard, 1991). Esta idea abarca a los sujetos mismos, pues cada uno ha de transformarse en un negocio al que debe agregar valor para poder asumir un rol operante dentro del sistema actual.

La noción de desarrollo ligada a dicho orden social, es justificada en la medida que supondría mayor bienestar colectivo pues se concibe la universalización (que tiene como meta que todos nos rijamos por un valor común mínimo) en tanto aquello que permite el crecimiento ilimitado y progreso prospectivo (Baudrillard, 2002b). Como consecuencia, la violencia es asociada a la pobreza, al subdesarrollo y el atraso, es decir, se percibe como un elemento que atenta contra "los placeres propios de la razón" (Saavedra, 2018: 17), valor insignia de la modernidad. Los grupos marginados fijados por el orden actual mundial a lo periférico, como grupos de desplazados, inmigrantes pobres o habitantes de calle, sin lugar claro en el capitalismo, son percibidos como pobreza caótica y señalados como peligrosos para el sistema mismo.

La condición de posibilidad para la emergencia de dicho sistema hipermoderno violento, en tanto excluye y margina, se encuentra ligada a la misma modernidad que le da paso. Como precedentes se pueden mencionar la conjunción entre la ciencia moderna y los valores de la revolución francesa que abonan el terreno en el que tiene lugar la revolución industrial (Baudrillard, 2002a). A partir de esta última, el planeta se transforma en un mercado global y se imponen como valores a la "libertad de competencia, la igualdad democrática y la fraternidad social" (Ramírez, 2018: 23). En este sentido, se puede mencionar que la instalación de un modelo de 
La violencia invisible de lo cotidiano y responsabilidad del profesional en ciencias sociales

sociedad produce todo el resto como desecho, a su vez, el discurso capitalista hace imposible el reconocimiento entre sujetos y culturas pues estos son convertidos en objetos equivalentes, evaluables, intercambiables, consumibles y desechables. La discriminación y la exclusión del otro, por medio de una violencia que enfrenta a todos contra todos, no es una desafortunada consecuencia de la globalización, sino el resultado lógico de un mundo regido por la competencia. Así, el sujeto liberado por la modernidad, se moviliza bajo el imperativo categórico capitalista que exige maximizar las ganancias sin importar las consecuencias y, por lo tanto, coincide con el sujeto imaginado por el Marqués de Sade, aquel que tiene derecho a gozar sin restricciones, como consecuencia, "el semejante desaparece como alteridad para pasar a existir en tanto instrumento" (Gallo, 2017: 61).

Ahora bien, para Lyotard (1997), el proyecto moderno es realizado hoy por la tecnociencia, pero desprovisto de los ideales de libertad, fraternidad e igualdad publicitados por la modernidad. En primer lugar, no somos tan libres si vivimos en ciudades que vigilan todos nuestros movimientos y que restringen libertades bajo el pretexto de la seguridad; donde la fraternidad es cada vez menos geniuda pues resulta más importante ocuparse de los propios intereses y en este sentido el otro es un medio para alcanzarlos o un obstáculo a eliminar; donde los derechos se transforman en negocios (de la salud o la educación por ejemplo); donde "hay cada vez más barreras sociales, segregación [...] estratificaciones” (Ramírez, 2018: 37), por lo que tampoco somos iguales en términos de acceso a oportunidades entre grupos poblacionales. Es decir, los valores "universales" (derechos humanos, libertad, cultura y democracia) mueren por efecto de la globalización del mercado. Los canales y plataformas de información hacen que dichos valores circulen como cualquier otra mercancía (Baudrillard, 2002b). Por eso, no sorprende el surgimiento de una nueva forma de violencia caracterizada por la desvalorización de lo humano y de la vida misma a la que se le asigna un valor de cambio. En Colombia lo ejemplifica el sonado caso de las ejecuciones extrajudiciales o falsos positivos, intensificados a partir del 2006, que involucran a miembros del ejército quienes, a cambio de ascensos e incentivos, participaron en el asesinato de civiles, en su mayoría jóvenes de sectores desfavorecidos, haciéndolos pasar como miembros de grupos armados al margen de la ley para mostrar la supuesta efectividad de las políticas de seguridad estatal (Gómez, 2020).

En este contexto, un arma clave para evitar que se declare la guerra (que históricamente ha amenazado al poder) es la disuasión (Baudrillard, 1978b). Como efecto de esta última, el planeta se reduce a un aparato logístico donde el ciudadano masificado, entregado por completo al autismo de las pantallas y el entretenimiento, deviene medida común (Daza - Zuela, 2017). Para Baudrillard (2002a), sin embargo, el juego no ha terminado, nos encontramos ante una tecnoestructura global omnipresente que, tras eliminar enemigos externos, ha quedado sola para ejercer su dominio, pero ahora se enfrenta a fuerzas heterogéneas que surgen de 
todas partes, que exigen ser escuchadas y que se niegan a ser homogeneizadas bajo el principio de equivalencia cultural producida por procedimientos industriales. Dicha reacción, asume la forma de singularidades que crean su propio juego e imponen sus propias reglas. Puede tratarse de manifestaciones colectivas de carácter étnico, religioso o lingüístico, o de arranques emocionales individuales que reaccionan de forma violenta, como lo ejemplifica el caso de Campo Elías Delgado, conocido como el psicópata de la masacre del restaurante Pozzeto que en los años 80 en la ciudad de Bogotá asesinó en un mismo día a 29 personas y es presentado en la novela "Satanás" del escritor bogotano Mario Mendoza (2002) (compañero universitario de Campo Elías) como síntoma de una ciudad despiadada volcada hacia el interés propio, la hipocresía, la apariencia, la clase social y el arribismo. Mendoza describe una ciudad que termina construyendo un monstruo al rechazarlo por no cumplir con los estándares de deseabilidad social. El personaje en la novela dice sentirse "[...] excluido, rechazado, como un leproso medieval, como si estuviera contagiado de una enfermedad que pudiera generar una pandemia" (103). Pero la heterogeneidad también puede manifestarse en forma de una rendición impotente ligada a un odio por sí mismo y al remordimiento. A este respecto, para Baudrillard (2006), el oscuro objeto del resentimiento y fuente de odios acumulados, es el exceso de comodidad, de disponibilidad universal, de logro definido e identidades impuestas contra las cuales lo heterogéneo se revela. Sobre esto volveré más adelante. Por ahora se mencionará un peculiar discurso fundado en la corriente de Pensamiento Positivo que nos invita a adaptarnos con una sonrisa al imperativo de productividad.

\section{La masificación del discurso de lo positivo y producción de sujetos desechables}

¿Acaso se puede ser otra cosa que no sea ser positivo? Teniendo en cuenta el discurso persistente que proclama en todas partes la felicidad y el optimismo como deber, la respuesta parece obvia, al fin y al cabo ¿Quién querría dar trabajo o socializar con una persona negativa? Esto es precisamente lo que cuestiona Barbara Ehrenreich (2012) en su libro Sonríe o Muere donde alude a la sospecha que le genera la corriente conocida como Pensamiento Positivo. Se trata de un movimiento originalmente filosófico-religioso, que ha ido impregnando diversos ámbitos como el de la salud, las empresas, las iglesias y, aunque marcando ciertos límites, a la misma disciplina psicológica. En el libro, la escritora señala una afinidad entre capitalismo (regido por la idea de progreso y competencia) y pensamiento positivo que rastrea siguiendo el post-calvinismo protestante en Estados Unidos. Un nuevo modelo conocido como Nuevo Pensamiento se rebela contra la antigua estructura

religiosa basada en la culpa y, en su lugar, instaura la idea de un Dios amoroso y de un mundo ya no adverso sino concebido como un espacio de suministro de 
La violencia invisible de lo cotidiano y responsabilidad del profesional en ciencias sociales

abundancia en el que el crimen, la enfermedad y la pobreza son atraídos por las mentes que perdieron su vibración cósmica con la generosidad y el amor. En lugar de cambiar la realidad (eso puede tardar toda la vida), se promueve modificar la percepción sobre esa realidad, se anima a pensar que lo que pasó es producto de nuestra actitud. Se nos dice que si superamos el resentimiento y pensamos en positivo tendremos una mentalidad ganadora. Además, la idea de que debemos deshacernos de las personas negativas de nuestra vida es repetida por oradores motivacionales, los otros no están ni para criticarnos ni para contradecirnos sino para aplaudir nuestras hazañas y para reafirmarnos. Para Ehrenreich (2002) se está produciendo un déficit masivo de empatía hacia quienes tienen dificultades pues sus problemas no son los nuestros.

Pero el mandato no es solo bloquear a personas negativas, sino a todo lo demás que pueda generar tristeza y ante lo que nada podamos hacer y que, por lo mismo, no vale la pena preocuparse. En cambio, la invitación es a retirarnos a un mundo interior donde todo sea aprobación y afirmación, para esto podemos ayudarnos acudiendo a técnicas como la visualización que incluye, como su nombre lo indica, representar visualmente nuestros objetivos que pueden ser financieros, románticos o incluso una cena en el restaurante favorito o las vacaciones de nuestros sueños. Respecto a este horizonte mencionado, la escritora norteramericana en cuestión (Ehrenreich, 2002), doctorada en biología, hace varios reparos. En primer lugar, insiste en que la mente no domina la materia. Por otro lado, plantea que el lugar tan perfecto (como uno quiere que sea) donde los sueños se cumplen siempre, equivale a un sitio dominado por una soledad espantosa. El resultado es un mundo "sin belleza, sin trascendencia y sin piedad" (176). Sin embargo, esto no quiere decir que la alternativa sea la desesperanza, que para la autora puede ser tan dañina como el excesivo optimismo. En cambio, resalta la importancia de salir de esa burbuja narcisista y megalómana que nos han vendido y propone manejar cierto nivel de sospecha pues la considera una actitud indispensable para sobrevivir como especie. Es más, según su criterio, la educación ha de impulsar el pensamiento crítico que no es igual a pensar positivamente pues el pensamiento crítico es escéptico por definición.

Por su parte, Baudrillard (1978a) destaca que precisamente en el instante en que se proclama con mayor vehemencia la autogestión (por demás bandera del pensamiento positivo), se empieza a constatar el desapego de la mayoría silenciosa. Para este autor, el creciente apoliticismo actual es síntoma de que estos grupos gregarios están adormecidos en sus responsabilidades ciudadanas por el confort narcisista del consumo y los media. No obstante, aclara que la masa indiferente no es producto del desierto cultural sino, todo lo contrario, de la saturación de intercambios. Esto es interpretado aludiendo a la seducción de las fuerzas productivas del mercado que lo invaden todo, inclusive el orden periodístico, antaño considerado como depositario de nuestra relación con lo verdadero. Las 
noticias llegan, se posan un instante, pero se desvanecen pronto en la superficie. Este orden es violento porque, así como con la circulación de noticias efímeras, produce todo como mercancía lista para ser consumida, desechada y olvidada. Por ejemplo, en la política, sus acontecimientos no tienen energía capaz de conmover, de ahí que se nos ofrezcan como una película de la que no somos responsables. Incluso las ideas revolucionarias se perdonan y se las puede exaltar, lo anterior en la medida que son vendidas "a plazos" en el mercado y por consiguiente deja de ser necesario que se realicen, nos podemos conformar con consumirlas en las redes (Baudrillard, 1981).

Aquí se puede dilucidar dónde está el milagro del sistema, pregunta que se hace el psicoanalista italiano Recalcati (2015) a la que responde que el fin último del sistema es la autorreproducción (repetición eterna de lo idéntico), en consecuencia, no alberga dialéctica social y no hace posibles ni la contradicción ni el cambio estructural. Tomemos por ejemplo los test y sondeos continuos considerados "formas de expresión" de las masas. Para Baudrillard (1978a), estos sondeos "devuelven a todas las preguntas que le son dirigidas una respuesta tautológica y circular"(135), no hay interlocutor pues son autorreferenciales. Este es el procedimiento por el que la masa se recluye en su silencio desapareciendo en tanto sujeto de la historia para reaparecer como dato estadístico. Ahí está el éxito de un sistema que, en su autoafirmación perpetua, transforma al hombre en una máquina acéfala destinada a cultivar la propia imagen a través del consumo. Cuando dicha máquina falla, la caída en el vacío resulta nefasta, como puede analizarse a partir de la actual difusión epidémica de la depresión. Aquel que no participa en la "movilización total" de la vida hacia su afirmación positiva, se vive a sí mismo como insignificante, como un desecho para la sociedad (como lo recuerda el caso de Campo Elías Delgado citado anteriormente). Dichos efectos no son ajenos a la idea narcisista que promueve construirse a sí mismo sin pasar por el Otro. Es decir, como carente de deudas simbólicas con el Otro del que se proviene (Recalcati, 2015) pues, al fin y al cabo, el infante nace biológicamente, pero son los otros los encargados de su entrada en la realidad simbólica del intercambio. De ahí la importancia de entender no solo que somos interdependientes, sino que el otro es la expresión de una diferencia radical que ha de ser respetada como tal.

\section{El potencial de activar lo heterogéneo, el vínculo social y lo simbólico en la práctica profesional}

Si prestamos atención a la noción de diferencia, nos encontramos con que remite al acto de diferir, es aquello que hace que dos cosas no sean la misma (Real Academia Española, 2001), lo que aplica para una persona pues diferimos en el tiempo, es decir, el sujeto no es idéntico a sí mismo pues no obedece a rasgos unitarios e invariables. Lo anterior se resalta con el fin de destacar la importancia de 
La violencia invisible de lo cotidiano y responsabilidad del profesional en ciencias sociales

no desactivar el potencial que supone la diferencia en tanto alteridad. En esta vía, para un sistema que se caracteriza por un goce sin límite, toda forma de vínculo se presenta como un obstáculo pues, en tanto incluyen a un otro, es decir a la alteridad, marca un borde, un punto de resistencia. El autor italiano Massimo Recalcati (2015) lleva este análisis al plano de la violencia destacando que cuando algo se opone a la máquina de goce, a la repetición de lo idéntico, la violencia irrumpe en escena para retirar cualquier obstáculo. Esto lo han constatado trágicamente en Colombia diferentes comunidades y sectores sociales que por suponer un límite respecto a la explotación de la tierra se han visto gravemente afectados por las consecuencias del conflicto armado interno (Centro Nacional de Memoria Histórica, 2013). En este sentido, una de las tareas más complicadas es la de saber renunciar a la violencia en nombre del reconocimiento del otro como prójimo, como ser singular, como diferencia absoluta. Lo que nos obliga a aceptar que "yo no lo soy todo, que mi vida no agota la del mundo ni la de los demás" (Racalcati, 2015: 117). Supone también sobrellevar aquello que el padre del psicoanálisis calificaba en su obra El malestar en la cultura (Freud, 1995) como una "frustración narcisista" que implica una renuncia a exigencias pulsiones sexuales y agresivas necesaria para reconocernos como miembros de una comunidad humana.

Pasando ahora a la práctica que atañe al terreno social a partir de lo hasta el momento señalado, podemos advertir con Lyotard (1997) que en nuestros tiempos el saber es algo de lo que uno "se apropia", siendo la relación de proveedor y usuario con el saber muy similar a la de productor y consumidor respecto a la mercancía. Ahora bien, la aparente suficiencia de este saber científico precede un "mundo de expertos" haciéndoles sentir que no tienen necesidad de otros saberes. En esto repara Baudrillard (1980) cuando se refiere a un estudio sobre una tribu de Samoa que aplica criterios materialistas y recomienda desarticularse en su actividad científica de cualquier tipo de ideología, pero, dice el autor, se soslaya que posiblemente la ideología vive implícita en eso conocido como actividad científica. En el caso referido, las contradicciones para el investigador aparecen por todas partes en el momento en que asombra percatarse de que "el primitivo" se niega a producir excedentes a pesar de que pueda producirlos. Baudrillard aquí denuncia el permitir que el orden de la producción domine todos los esquemas comprensivos, lo que nos colocaría ante un acto de violencia teórica por no decir política pues las disciplinas sociales pueden terminar siendo cómplices de acciones que buscan, bajo las mejores intenciones, eliminar estos obstáculos sociales y culturales que "obstruyen" la posibilidad de abrir el camino hacia el crecimiento tecnológico moderno de los llamados "pueblos primitivos", es decir, a la transformación de lo diferente en lo idéntico.

Finalmente, cabe destacar que el psicoanálisis encuentra la idea de identidad y de comprensión sospechosas, precisamente porque, en las categorías utilizadas con el fin de comprender y categorizar a alguien como perteneciente a una clase, se pierde 
lo que cada quien tiene por producir sobre el síntoma o el malestar que le concierne (Lacan, 2017). Por su parte, desde la filosofía, Paul Ricoeur (1996), en lugar de buscar un principio de unidad en criterios causales y de continuidad de estados, abre paso a un modelo basado en la identidad narrativa. En este sentido, para saber quién es alguien, más que apuntar a características esencialistas, se cuenta una historia sobre esa persona. La narración emerge como una forma de producción de sentido en la que no hay identidad hasta que no haya un relato significativo para el sujeto portador de esa identidad.

A partir de estas puntualizaciones, más que buscar en la práctica un núcleo de comprensión explicativo utilizando categorías impuestas desde el exterior, se advierte que las acciones, deseos y valores cambian de modo continuo en función, por ejemplo, de un giro en el diálogo que puede tener lugar en procesos de escucha y de acompañamiento comunitario del profesional. De ahí la importancia de resaltar el enfoque Acción sin Daño (Rodríguez, 2010) desde el cual se advierte que a veces las soluciones propuestas por el investigador suman problemas a la comunidad con la que trabaja pues dichas soluciones se han planteado desde afuera por el "experto" bajo la premisa de que todos los grupos son iguales y, en consecuencia, es posible replicar modelos y seguir protocolos de intervención que han sido diseñados para otras latitudes.

En esta línea, resulta revelador lo dicho por Bustamante (2013) cuando advierte que "las diferencias no están ahí para hacer listados y clasificaciones con ellas. A veces encarnan decisiones y, en tanto tales, se pueden asumir" (8), en otras palabras, toda intervención tiene efectos, la cuestión está en interrogarse como profesional al respecto, tomar posiciones, así como estar dispuesto a asumir las consecuencias. Entonces, a diferencia de lo que interpretan prácticas permeadas por la lógica de la economía política con su obsesión por la repetición de una identidad, se propone un trabajo con sujetos y comunidades más en la vía de narrativo o de lo poético, pues como resalta el escritor francés al que me he referido en varias oportunidades en este texto, es en lo poético donde las identidades son sacrificadas (Baudrillard, 1992), lo poético adquiere su fuerza y poder revolucionario precisamente donde menos se lo espera, no en la uniformidad sino en el diferimiento.

De ahí la importancia de problematizar la idea según la cual la paz es ausencia total de violencia gracias a la unificación de los contrarios, es más, la violencia se concibe como una relación social permanente que puede tener salidas distintas no siempre destructivas. Siguiendo esta conjetura, lo que hace factible que no todo malestar subjetivo devenga en un acto violento como el de Campo Elías Delgado implica una tramitación del mismo por medio de recursos simbólicos. Por lo tanto, el objetivo de poner en marcha un proceso de acompañamiento en el campo de lo social, no es suprimir las relaciones conflictivas a través de herramientas puestas en acción por el profesional para generar cambios que aseguren la conformidad voluntaria de los intervenidos hacia ciertas formas "deseables" de ver el mundo. En 
La violencia invisible de lo cotidiano y responsabilidad del profesional en ciencias sociales

su lugar, por medio de metodologías como la propuesta desde el enfoque Investigación Acción participativa-IAP, cuyo referente principal en sociología es Orlando Fals Borda (Colmenares, 2012), se trata de permitir la construcción de un saber, junto con la comunidad implicada, sobre unas lógicas propias respecto a violencias que acontecen en un contexto singular y que a pesar de no siempre coincidir con el "deber ser social", se vinculan a modos de vida que responden a las maneras que las comunidades han encontrado, a partir de los recursos e historia particulares, para lidiar con el conflicto. Esta metodología invita a un fortalecimiento de lo simbólico pues aunque la violencia sea inextinguible, si es posible tramitarla y puede ser sustituida por medio del debate, el deporte, el arte y otras formas de ejercer una fuerza sobre el otro que no lo eliminan sino que lo construyen como diferencia que, en vez de completar, descompleta (Gallo, 2017). Esta tarea implica separarse del yo para ir hacia el otro y hacer con él una alianza que, como lo señala la filósofa norteamericana Judith Butler (2010), no exige estar de acuerdo en "todas las cuestiones del deseo, creencia o autoidentificación" (55). Supone, al contrario, darles cabida a ciertos tipos de antagonismo en curso entre sus participantes, "valorando las diferencias persistentes y animadoras como signo y sustancia de una política democrática radical" (56).

En este punto, retomo la inquietud señalada en el título sobre el quehacer profesional, pues este, al tener efectos políticos, sociales y comunitarios sobre quienes recae, implica asumir una responsabilidad. A este respecto, la IAP, permite pensar en un modo de proceder distinto a la lógica racionalista centrada en la función y posibilita en cambio no solo pensar los efectos de la propia práctica, sino también, permite introducir una mediación simbólica para la construcción de versiones inéditas sobre la implicación de cada uno de los miembros de un grupo en eso que causa malestar y así posibilitar procesos de transformación social, de producción de conocimiento, análisis de recursos propios y aporte a la tramitación de las manifestaciones de violencia. Cabe anotar que no todas las singularidades que reclaman ser reconocidas se manifiestan por medio de actos violentos, las madres de la Plaza de Mayo en Argentina y las madres de Soacha en Colombia han resistido a través de actos simbólicos con los que deslegitiman el veredicto de muerte a lo heterogéneo enunciado por el Estado. Vale la pena que estos actos de palabra sean escuchados en su singularidad más radical.

Finalmente, me gustaría mencionar los Montes de María, una región del Caribe colombiano donde, entre 1985 y 2017, han ocurrido 117 masacres, más de tres mil asesinatos políticos y doscientos mil desplazamientos forzados (Centro Nacional de Memoria Histórica, 2014). Pero no deseo ampararme en la cifra fría que no genera mayor impacto ni garantiza que lo sucedido no se repita, menos aún en un país que se ha acostumbrado a recibir el conflicto como piezas de información emitidas todos los días por los noticieros, este exceso de información satura y produce indiferencia. Tampoco deseo destacarla como una tierra de víctimas, sino como un 
lugar en donde ante el silencio al que fueron condenados por otros, le apostaron a la invención de estrategias para narrarse ellos mismos, desde su territorio, de otras maneras. Este fue el propósito del colectivo de Comunicaciones de los Montes de María que funda el festival de cine comunitario más importante del país como una estrategia para que la gente volviera a salir a las calles; que hace uso de la radio, del arte y del canto como vehículo para narrar; que después de un largo proceso de repensar su territorio y sus historias de vida sienta las bases del primer museo de carácter itinerante reconocido como un espacio de memoria y nombrado por un ave cantora de la zona convertida en símbolo, el Mochuelo. El museo, como el ave, se da a la tarea de recorrer las comunidades que conforman el territorio. Estas plataformas de reparación simbólica constituyen un homenaje a los muertos que emerge como iniciativa de la sociedad civil (los sobrevivientes) y los implica más allá del guiño instantáneo, simplista y poco efectivo de la noticia compartida en las redes, son sobrevivientes que van más allá de la muerte.

\section{Conclusión}

La búsqueda del beneficio propio parece ser la consigna de la alianza entre economía y ciencia que hoy no deja de tener consecuencias en lo social implicado, por ejemplo, en conflictos armados como el colombiano. En este sentido, la guerra se ha convertido en una estrategia más de mercado donde impera el interés personal y donde la vida y el vínculo con el otro se mercantilizan. Esta mercantilización de lo humano, propia de nuestra época, retorna también en lo social cotidiano en forma de odio expresado en una violencia hacia aquellos marginados de las posiciones sociales operantes. Para Baudrillard, esta violencia cotidiana se gesta dentro del sistema mismo y emerge de la prohibición del conflicto que tiende a eliminar las raíces del mal a fuerza de discursos profilácticos como el promovido desde la corriente del Pensamiento Positivo. En otros términos, se trata de la violencia ejercida por un sistema que hostiga cualquier forma de negatividad o de singularidad y que, con la pretensión de considerarse universalmente válido, produce funciones modelo bajo la lógica de "lo mismo". En este proceso de homogeneización constante, también el sujeto es sacrificado en la medida que se hace de él un objeto de producción para el mercado, es decir, la alteridad irreductible (la que hace la diferencia) termina siendo la prohibición mayor en la cultura de la igualdad, del empoderamiento y el crecimiento personal.

No obstante, la alteridad no deja de manifestarse ante dicho intento de unificación en nombre de valores universales. El resentimiento y el odio, como lo ejemplifica el caso de Campo Elías Delgado, proliferan junto con la explosión de subjetividades que solicitan el derecho a ser reconocidas. Ahora bien, cuando el ser humano es tomado por medio de modelos y protocolos educativos preestablecidos como un individuo factible de ser programado a semejanza de la máquina, se 
La violencia invisible de lo cotidiano y responsabilidad del profesional en ciencias sociales

incentiva el aburrimiento y la devaluación del sujeto. Por lo tanto, desde una perspectiva que entiende que los humanos no dependemos de procesos masivos sino singulares, en lugar de proporcionar a las colectividades herramientas estandarizadas de adaptación y desarrollo de competencias, la invitación al profesional en Ciencias Sociales es a inventar estrategias que posibiliten a cada uno conquistar un deseo de saber, así como renunciar a modos de satisfacción narcisista que separan al ser humano del vínculo social. En esta vía, la Investigación Acción Participativa y el Enfoque Acción sin Daño tienen la virtud metodológica de permitir reconocer en la comunidad a un actor social comprendido por subjetividades con capacidad de participación activa.

\section{Referencias bibliográficas}

Baudrillard, J. (2006). Violencia política y violencia transpolítica. En: Amaya, A. (ed.) Los límites de la estética de la representación. Bogotá: Universidad del Rosario.

Baudrillard, J. (2002a). La ilusión vital. Madrid: Siglo XXI.

Baudrillard, J. (2002b). La Violence du Mondial. En: Baudrillard, J. Power Inferno. Paris: Galilée. 62-83.

Baudrillard, J. (1992). El intercambio simbólico y la muerte (1976). Caracas: Monte Ávila Latinoamericana.

Baudrillard, J. (1991). Después de la orgía. En: Baudrillard, J. La transparencia del mal. Ensayo sobre los fenómenos extremos. Barcelona: Anagrama. 9-19.

Baudrillard, J. (1981). El sistema de los objetos. Ciudad de México: Editorial Siglo xxi.

Baudrillard, J. (1980). El espejo de la producción. Barcelona: Gedisa.

Baudrillard, J. (1978a). A la sombra de las mayorías silenciosas. Barcelona: Kairós.

Baudrillard, J. (1978b). Cultura y simulacro. Barcelona: Kairós.

Bustamante, G. (2013). Sujeto Sentido y Formación. Bogotá: Editorial San Pablo.

Butler, J. (2010). Marcos de guerra. Barcelona: Paidós.

Centro Nacional de Memoria Histórica (2014). El primer vuelo del Mochuelo. Asequible en: https://centrodememoriahistorica.gov.co/el-primer-vuelo-del-mochuelo/, fecha de consulta: 20-07-2021.

Centro Nacional de Memoria Histórica (2013). !Basta ya! Colombia: Memorias de guerra y dignidad. Informe general grupo de memoria histórica. Bgotá: Imprenta Nacional.

Colmenares, A. (2012). Investigación-acción participativa: una metodología integradora del conocimiento y la acción. Voces y Silencios. Revista Latinoamericana de Educación, III(1). 102-115. 
Daza, G. - Zuela, M. (2017). La política de la guerra sin estado de guerra. Nomadas (Colombia), vol. 8. 104-113.

Ehrenreich, B. (2012). Sonríe o muere: la trampa del pensamiento positivo. Madrid: Turner. Freud, S. (1995). El malestar en la cultura. En: Freud, S. Obras Completas Tomo XXI. Buenos Aires: Amorrortu.

Gallo, H. (2017). Psicoanálisis e intervención psicosocial. Medellín: Universidad de Antioquia.

Gómez, J. (2020). Antecedentes históricos sobre los "falsos positivos" en Colombia. En: Barbosa, G. - Ciro, A (ed). Garantía de no repetición: una contribución a la justicia transicional. Bogotá: Universidad Externado de Colombia.

Lacan, J. (2017). Seminario 3 (955-56). Las psicosis. $1^{\mathrm{a}}$ ed. $25^{\mathrm{a}}$ reimp. Buenos Aires: Paidós.

Lyotard, J. (1997). La condición postmoderna. Madrid: Cátedra.

Lopera, J. - Ortiz, J. - Ramírez, C. - Zuluaga, M. (2010). El método analítico. Medellin: CISH-Universidad de Antioquia.

Mendoza, M. (2002). Satanás. Bogotá: Planeta.

Mojica, C. P. (2015). Violencia transpolítica en el cuento colombiano: análisis de una ciudadanía emergente en "Álbum de billetera", de Rodrigo Parra Sandoval. Estudios de Literatura Colombiana, Issue 36. 145-160 .

Montesinos, D. (2002). El poder y los signos. Baudrillard y la incertidumbre de la crítica. Valencia: Universitat de Valencia.

Ramírez, M. (2018). Conflicto armado y subjetividad: Nuevos ensayos de psicoanálisis aplicado a lo social. Primera ed. Buenos Aires: Grama Ediciones.

Real Academia Española (2001). Diccionario de la lengua española [versión 23.4 en línea]. Asequible en: https://dle.rae.es, fecha de consulta: 29-07-2021.

Recalcati, M. (2015). Ya no es como antes. Elogio del perdón en la vida amorosa. Barcelona: Anagrama.

Ricoeur, P. (1996). Si mismo como otro. México: Editorial Siglo XXI.

Rodríguez, A. (2010). El enfoque ético de la acción sin daño. En: Bello, M (ed.). Programa de Iniciativas Universitarias para la Pazy la Convivencia (PIUPC). Bogotá: Universidad Nacional de Colombia.

Saavedra, A. (2018). Cuando la paz duele. Reflexiones en torno al genocidio contra la Unión Patriótica en Colombia. Negonotas Docentes, Issue 11. 15-26. 